Revista Científica

\title{
El teatro y los valores en educación
}

\author{
Theater and values in education \\ Teatro e valores na educação
}

ARTÍCULO GENERAL

\section{Segundo Dario Servan Picon \\ darioservanpicon@gmail.com \\ https://orcid.org/0000-0002-8160-0408 \\ Escuela de Postgrado de la Universidad César Vallejo}

Recibido 12 de Setiembre 2021 | Arbitrado y aceptado 12 de Setiembre 2021 | Publicado en 12 de Octubre 2021

\section{RESUMEN}

Esta investigación tiene como objetivo determinar si el teatro o arte dramático contribuye como disciplina artística en la mejora de los aprendizajes en el campo educativo sobre todo en la práctica y el desarrollo de actitudes relacionadas a una cultura de valores. Se propone este estudio como base para la reflexión sobre una educación en valores, partiendo del aprendizaje del arte dramático del que se destaca un doble sentido estético y ético-formativo.

Es posible también contemplar en este estudio una reflexión validada sobre la posibilidad o no de plantear una educación en valores apoyada en el aprendizaje del teatro independientemente de cualquier ideología o creencia. La metodología del estudio se sustentará en la búsqueda y revisión de la bibliografía y los estudios previos sobre e papel del teatro en el campo educativo así como sus aportes en el desarrollo de actitudes y valores. Esto como base para una reflexión crítica sobre la práctica de la educación en valores específicos en el aula.

Los resultados de estos análisis previos sobre nuestro tema a investigar nos servirán para acercarnos de un modo objetivo y realizar la discusión de estos resultados en la búsqueda de una postura. Las conclusiones con la que iniciamos esta investigación apuntan primero, a que existen carencias en la educación actual, como es el de establecer vínculos afectivos motivadores para la educación en valores. En segundo lugar, se puede afirmar que con el uso de las artes y sus diferentes estrategias en la escuela como referentes didácticos se puede facilitar la empatía necesaria para la vivencia de los valores en el aula. Sin duda las conclusiones finales de esta investigación reafirmaran una postura conveniente frente al teatro y el rol importante que cumple en el campo educativo.

PALABRAS CLAVE: Teatro, Arte, Valores, Educación

\section{ABSTRACT}

This research aims to determine if theater or dramatic art contributes as an artistic discipline in improving learning in the educational field, especially in practice and the development of attitudes related to a culture of values. This study is proposed as a basis for reflection on an education in values, starting from the learning of dramatic art from which a double aesthetic and ethical-formative sense stands out.

It is also possible to contemplate in this study a validated reflection on the possibility or not of proposing an education in values supported by the learning of theater regardless of any ideology or belief. The methodology of the study will be based on the search and review of the bibliography and previous studies on the role of theater in the educational field as well as its contributions in the development of attitudes and values. This as a basis for a critical reflection on the practice of education in specific values in the classroom.

The results of these previous analyzes on our topic to be investigated will help us to approach in an objective way and carry out the discussion of these results in the search for a position. The conclusions with which we began this research point first to the fact that there are shortcomings in current education, such as establishing motivating affective links for education in values. Secondly, it can be affirmed that with the use of the arts and their different strategies in school as didactic references, the necessary empathy for the experience of values in the classroom can be facilitated. Undoubtedly, the final conclusions of this research will reaffirm a convenient position towards theater and the important role it plays in the educational field. KEY WORDS: Theater, Art, Values, Education

\section{RESUMO}

Esta pesquisa tem como objetivo verificar se o teatro ou a arte dramática contribui como disciplina artística na melhoria da aprendizagem no campo educacional, principalmente na prática e no desenvolvimento de atitudes relacionadas a uma cultura de valores. Este estudo é proposto como base para a reflexão sobre uma educação em valores, a partir da aprendizagem da arte dramática, da qual se destaca um duplo sentido estético e ético-formativo.

Também é possível contemplar neste estudo uma reflexão validada sobre a possibilidade ou não de se propor uma educação em valores sustentada na aprendizagem do teatro independentemente de qualquer ideologia ou crença. A metodologia do estudo será baseada na pesquisa e revisão da bibliografia e estudos anteriores sobre o papel do teatro no campo educacional, bem como suas contribuições no desenvolvimento de atitudes e valores. Isso serviu de base para uma reflexão crítica sobre a prática da educação em valores específicos em sala de aula.

Os resultados dessas análises prévias sobre o tema a ser investigado nos ajudarão a nos aproximar de forma objetiva e realizar a discussão desses resultados na busca por uma posição. As conclusões com as quais iniciamos esta pesquisa apontam, em primeiro lugar, para o fato de que existem lacunas na formação atual, como o estabelecimento de vínculos afetivos motivadores para a educação em valores. Em segundo lugar, pode-se afirmar que com o uso das artes e de suas diferentes estratégias na escola como referências didáticas, pode-se facilitar a empatia necessária para a vivência dos valores em sala de aula. Sem dúvida, as conclusões finais desta pesquisa reafirmarão uma posição conveniente em relação ao teatro e o importante papel que desempenha no campo educacional.

PALAVRAS-CHAVE: Teatro, Arte, Valores, Educação 


\section{INTRODUCCIÓN}

Segundo Dario Servan Picon

Los valores permanentemente ha sido un tema de discusión en educación, cuya causal importante viene de la sociedad que construye un sistema de normas y jerarquías cambiantes en el tiempo. Las instituciones educativas y la sociedad tienen dificultades para educar en valores en torno a la doctrina y la particularidad con que se implementan estos valores. En ese panorama urge la necesidad de no solamente enseñar valores sino también evaluarlos. La falta de valores puede ser tomada como la principal causa la degradación de la familia como estructura, es decir ya no es la familia el lugar donde confluyan actitudes y valores.

Es probable que existan otros factores como la influencia de los medios de comunicación y otros. Es en este panorama que nuestra mirada se fija en la práctica dramática y en el como a través de sus bondades se puede educar en valores, Empoderar esta disciplina del arte en el plano educativo en la formación de actitudes en un marco de una sana convivencia basada en valores son uno de los objetivos de este estudio.

Este estudio también pretende abordar cuál es el nivel de influencia de las actividades dramáticas o la práctica teatral en el desarrollo de valores en los estudiantes, así como los aportes del teatro a la educación actual. Consideramos en este estudio, que el teatro como actividad artística potencia la expresividad del estudiante a la vez que le hace ver que en la libertad de estas expresiones hay opiniones además de la suya igualmente respetables. Por ello el teatro es la actividad artística que conjuga estas dos posturas (escuchar y exponer), que son el eje de la convivencia y la ciudadanía.

\section{MÉTODO}

Este artículo forma parte de un estudio en proceso sobre la aplicación de un taller de teatro para la práctica de valores. El fundamento es que el teatro como estrategia pedagógica es potencialmente útil en la formación y desarrollo de valores en educación. En particular en este artículo, se presentan la síntesis de la búsqueda en bases de datos, acerca del tema: es decir nivel de relación entre educación y valores, arte y educación y el teatro con los valores. Los criterios para seleccionar la información de las fuentes fueron los siguientes: precisar las principales tendencias encontradas en relación al estudio. Ordenar los conceptos básicos que fundamentan la relación según los temas tratados, identificar los objetivos y conclusiones y, señalar las evidencias de investigación en cada una de las tendencias identificadas. En la búsqueda de la información se tuvo en cuenta en primer lugar, los libros electrónicos, los trabajos de 
Segundo Dario Servan Picon

investigación de maestría y tesis de Doctorado, realizadas sobre el tema, y en segundo lugar, al análisis de los artículos encontrados sobre este tema en las Bases de datos: scielo, Dialnet, Ebsco, etc. Para el inventario y organización de la información, así como para la referencia bibliográfica utilizamos Mendeley aplicativo que nos garantiza la fiabilidad y validez de los estudios encontrados. En el artículo se reseñan en primer lugar los conceptos que orientan el estudio desde una perspectiva general, en esta parte ponemos en evidencia los fundamentos conceptuales en los que se basan las tendencias encontradas. En segundo lugar, se presentan aquellos trabajos que ofrecen evidencias de investigación acerca de estos fundamentos conceptuales. Por razones de espacio no se incluyen aquí los resultados encontrados acerca de las metodologías usadas en la indagación de cada una de las tendencias establecidas cuando se analizó la información proveniente de las bases de datos acerca del tema.

\section{DESARROLLO Y DISCUSIÓN}

\subsection{EDUCACIÓN EN VALORES}

Sobre los valores en educación, (Touriñan, 2014) considera que estos se fundamentan en "el aprendizaje y la construcción de experiencias axiológicas que direccionaran un proyecto de vida del estudiante y/o individuo.” (Touriñan, 2014, p. 10). Por lo tanto, sin la práctica y la vivencia de los valores es imposible que los seres humanos se desarrollen completamente; tampoco es posible que se prepare un proyecto de vida y afronte a las interacciones de una sociedad justa y próspera. En función de los valores definimos lo bueno y malo de nuestras acciones como seres humanos Adoptar y desarrollar estos valores están sujetos a variables como la educación, la experiencia y la cultura.(Corzo, 2004)

Por otro lado, existe una educación no estandarizada u oficial, es la educación social, la cual puede ser vista como complemento a la formación recibida en las escuelas, esta tiene el propósito de educar en la convivencia para afrontar los diversos desafíos actuales y a futuro. Esto quiere decir que la educación social contribuye a la adquisición de valores, competencias, experiencias y voluntades individuales y colectivas. Desde este punto de vista, podemos encontrar una conjunción entre el arte dramático y la educación social, puesto que ambos están compuestos por reglas, métodos, contenidos, entre otros.

Sin embargo, cuando se reúnen las posibilidades pedagógicas que ofrece con los objetivos de la educación social, se produce una dinámica bastante interesante que 
Segundo Dario Servan Picon

permite a los enseñantes aprovechar una multitud de ocasiones para hacer un teatro vivo y una enseñanza activa y participativa; porque el teatro tiene mucho en común con los objetivos de la educación social. (Laferriere, S/N, p. 13)

La educación, estandarizada, basada en los lineamientos de una política pública, es decir una educación formulada por el Estado, a través de sus órganos de control, como el Ministerio de Educación, las Direcciones Regionales de Educación (DRE), las Unidades de Gestión Local de la Educación (UGEL), entre otros, los cuales tienen la finalidad de crear y supervisar el contenido educativo. El modelo educativo oficial se basa en la enseñanza de contenido basado principalmente en los parámetros leer, escribir, sumar y restar, y por otro lado, demuestra deficiencias para educar en valores. Cada vez más a menudo, son noticia conflictos al interior del aula, alumnos ${ }^{1}$ agreden a profesores o profesores ${ }^{2}$ ejercen en exceso el castigo sobre sus alumnos. Entonces, surge la necesidad de reformar el contenido de la educación, desde una base de valores, los cuales deben ser enseñados con la finalidad de ser puestos en práctica. Nos preocupa la desintegración de familia, la cual puede tener la pérdida de valores. Por lo tanto, buscar alternativas que permitan experimentar valores y ponerlos en práctica termina siendo gravitante, en este sentido, el arte puede compensar y complementar lo que a la educación formal le falta.

Frente a esta educación, aparecen sistemas educativos, con sus propios contenidos, en paralelo, a nivel de familia, de barrio, de trabajo, la iglesia, entre otros grupos socioculturales. Aparece pues un nuevo paradigma, dicotómico-complementario, basado en nuevos escenarios de enseñanza, variabilidad de educadores y educandos, diversos canales de comunicación, entre otros. En base a ello, aparece el arte como espacio y contenido educativo.

[...] Y entre las bases para construir este nuevo modelo educativo se concretan: complejidad, educador educando, didáctica centrada en el aprendizaje, inteligencias múltiples, sujeto colectivo, educación como diálogo abierto, ciudadanía, creatividad, cambio de percepciones y valores, sostenibilidad, interdependencia y conectividad, interdisciplinariedad y transversalidad, multiculturalidad, nuevas tecnologías y calidad con equidad. (Motos, 2009, p. 3)

\footnotetext{
1 Ver alumno agrede a profesor con una papelera. https://www.lasexta.com/programas/arusitys/virales/la-brutal-agresion-de-un-alumno-a-un-profesorcon-una-papelera_201912115df0befb0cf2c45df3179ebe.html

2 Ver profesor golpeó a 18 alumnos por no cumplir con la tarea en Arequipa. https://peru21.pe/peru/profesor-golpeo-18-alumnos-cumplir-tarea-478476-noticia/
} 
Segundo Dario Servan Picon

Por otro lado, el arte constituye la plataforma o medio de comunicación, por lo tanto, con posibilidades de ser un medio efectivo de enseñar y enseñar con valores, el arte es, por lo tanto, una creación-producción humana donde se representan situaciones cotidianas, o productos de la libertad de imaginación. (Paoli, 2010)

Entonces pues, hay que mirar en el arte las posibilidades de motivar, educar, explicar y fomentar una postura crítica de los educandos. Fomentar una cultura de valores, que suplemente la formación en el sistema educativo formal. Un punto de partida debe ser la propia escuela, pues no podemos dejar de lado las instituciones estatales, implementar cursos de arte, donde los educandos puedan expresarse, aprender y ejercitar los valores. Las artes escénicas pueden ser el camino a la manifestación de inquietudes y a la represión social. El teatro puede escenificar, dar voz, exhibir problemáticas como el bullying, y ser el inicio de la solución. Paoli (2010) expresa su esperanza en una sociedad más saludable, a través del abanico de posibilidades que ofrece el teatro.

El horizonte que incorpora valores humanos a la mirada permite trazar modos más edificantes de proyectar, de orientar el camino, de tramar periplos, modos de imaginar que fácilmente entusiasman. (Paoli, 2010, p. 15)

Los valores para cada generación están previamente establecidos con normas, ideales, leyes, preceptos del que se adueña a través de la educación y por ende esto le permite integrarse a la sociedad. La ONU (2000) en el marco de acción para la educación en valores en la primera infancia, contempla el respeto a sí mismo y a los demás, apoyando la cooperación y las habilidades para resolver conflictos; así como el aprecio a la diversidad, conciencia global y educación multicultural; como bases fundamentales ante una posible educación en valores desde la niñez. Según Ortiz (2003), cada sociedad, en un momento determinado de su historia, selecciona del sistema general de valores aquellos que considera más adecuados para satisfacer las necesidades sociales, siendo la escuela la institución encargada de su transmisión y desarrollo, por medio de la actividad educativa que se desarrolla en su seno.

Son las escuelas, instituciones muy importantes, que tienen función de transmitir conocimientos, así como, formar a los alumnos en valores. La herramienta más poderosa para lograr una sociedad saludable, donde se puedan desarrollar las futuras generaciones en un ambiente donde se pueda ser libre de ejercitar sus derechos con responsabilidad, es el sistema educativo, ahí es donde se forman ciudadanos. Sin embargo, por diversas causas, las escuelas no terminan de cumplir con dichos objetivos 
Segundo Dario Servan Picon

en su totalidad. Aparecen muchas veces como entidades, represivas, las cuales van a generar en los alumnos la necesidad de expresarse en otros ámbitos.

Hay pues una dicotomía entre el modelo de educación y el tipo de educando. Por lo que se requiere enfocarnos en fomentar a través de todos los medios posibles, en enseñar, fomentar, y practicar valores. Muchas veces se ha de buscar valores en otras esferas de la sociedad, y actualmente los valores son de carácter global y se puede afirmar que descubrir los valores sólo es posible a quien mira el mundo en forma positiva y encuentra que las cosas y los seres vivos tienen, porque que ha comprendido que todo lo que existe "existe por algo y para algo"; que cualquier ser, por insignificante que sea, tiene sentido y una razón de ser, es decir es "valorable" (Tierno, 2007)

\subsection{ARTE Y EDUCACIÓN}

La creencia popular de la psicología consideraba que lo importante en el arte es el placer, y el disfrute, es decir el arte visto como una experiencia básicamente emocional (Arnheim, 1997). En estos tiempos es imposible seguir pensando de esa manera, sabemos que cambió y a la luz de la educación el arte refuerza cada proceso de enseñanza y aprendizaje. Uno de los problemas que presenta el arte con respecto a las aulas, es que se concibe como un talento innato y no como una materia en la que entran procesos mentales e intelectuales, ya que al fin y al cabo es la mejor herramienta para educar la mirada. Gardner considera el arte como una actividad de la mente.

(Gandarillas 2015)

Los investigadores en educación reconocen la importancia de las artes en el currículo. El arte no implica el solo hecho de cumplir una tarea, a cambio nos ofrece conexiones de experiencias y sentimientos así como desarrollo habilidades propensas a ser valoradas como tal. El arte, según el Minedu (2018), en educación refuerza las identidades y los valores personales y colectivos, y, consecuentemente, contribuye a un diálogo intercultural que nos permite una mejor convivencia de ahí que las artes en el currículo actual debe cambiar los preceptos de la enseñanza basada en la repetición o adquisición del conocimiento, ya que a través del arte se utiliza el conocimiento con fines intelectuales, cognitivos y actitudinales. Gardner (1994) afirma que modular de un modo eficaz los valores de la cultura, los medios disponibles para la educación en las artes y para la evaluación, así como los particulares perfiles individuales y de desarrollo de los estudiantes a educar son los desafíos de la educación artística.

Entender el arte paralelamente a lo que propone Gardner en su forma estructural solo es posible a través de la espontaneidad, arte es entonces dejar fluir los sentimientos 
Segundo Dario Servan Picon

que guardamos en lo más profundo, Toda expresión en arte debe estar unida a la forma de percibir las cosas externas de cada persona, ya que así, podremos expresarnos de una manera espontánea e individual que nos conecte con el exterior. Al respecto Touriñán (2018) aclarando un poco más el rol del arte en la educación explica que el arte es un producto cultural de la capacidad creativa del hombre. Este potencial creativo y su desarrollo tienen a la escuela como escenario ideal.

\subsection{EL TEATRO Y LA EDUCACIÓN EN VALORES}

El teatro utiliza como forma de expresión escrita el diálogo, este se viste con la voz del actor que le imprime los matices para acariciar el oído del espectador.(Garzón, 2015) .El teatro en su término más genérico es una disciplina del arte que consiste en representar hechos reales o imaginarios sobre un escenario o un espacio preparado previamente, en el intervienen actores que representan personajes a través de diálogos y una situación dramática. En este acto de representar y actuar quien lo practica transmite valores intrínsecos de esta disciplina como son la libertad y la verdad. A propósito, Frías (2009) nos dice que el trabajo de la interpretación en el teatro consiste en la búsqueda constante de los valores de la verdad y la autenticidad. Sin embargo para quienes no han recibido formación pedagógica dramatización y teatro son lo mismo y los relacionan con textos y espectáculos teatrales, actores, autores, ensayos, vestuario, escenarios, etc. En ese sentido es preciso tener en claro que Teatro y Dramatización no son la misma cosa (Motos, 2009).

Para Domínguez (2020), la dramatización se centra en la práctica lúdica tomando como herramienta la actividad teatral donde a juzgar es importante el proceso y desarrollo de actitudes y habilidades del estudiante más allá de un resultado producto que busca básicamente el teatro formal. Motos ( 2008) nos dice que hacer dramatización es poner a los participantes en situación de desplegar y practicar su potencialidad creativa. Partiendo de esta premisa se hace una relación de las habilidades de creatividad dramática en los distintos niveles educativos. El teatro como estrategia pedagógica promueve el desarrollo de ejercicios dramáticos que posibiliten la libre expresión de los estudiantes, como parte de la necesidad de comunicarse, crear, recrear e imitar episodios de su vida cotidiana, desde un punto de vista formativo (MINEDU, 2010). Veamos, por ejemplo, las escenificaciones del grupo Les Luthier ${ }^{3}$, puestas en escena con contenidos muy diversos, que abordan temas de filosofía, ética, moral, valores, leyes, literatura, matemáticas, historicidad, y sobre todo múltiples maneras de

${ }^{3}$ Véase, La Gallinita dijo Eureka. En: https://www.youtube.com/watch?v=VENkNeay3jE\&t=194s 
Segundo Dario Servan Picon

creatividad. Este es un ejemplo de lo beneficioso puede llegar a ser el teatro para los estudiantes. Una forma de ver el conocimiento formal, de manera lúdica.

Las actividades dramáticas como parte del teatro exploran las diferentes habilidades del estudiante, incidiendo sobre todo en las distintas formas de comunicación, es interesante y valioso el aporte de las actividades dramáticas en el desarrollo de la libre expresión del estudiante por las variantes que esta presenta. Estas actividades se pueden agrupar de la siguiente manera: Teatro de títeres, pantomima. Improvisaciones, danza creativa, drama creativo, sociodrama, juegos y ejercicios dramáticos, teatro escolar, teatro formal, teatro callejero, entre otros. En la escuela los objetivos del teatro y la dramatización no es formar futuros artistas dramáticos sino despertar al estudiante para que tome conciencia de sí mismo, de los otros y del mundo que le rodea, es decir, tiene como meta el proceso de crecimiento personal y grupal a través del juego y ejercicio teatral (Motos, 2014). En base a este concepto, teatro como expresión cultural y personal, el cual se desarrolla en ámbitos de educación formal, debemos considerar también, la proyección social que tiene hacer teatro escolar. Esto puede ser de gran valor para transformar la sociedad, iniciando por el área de influencia de la escuela. Hablamos de hacer teatro para la comunidad en extramuros, las contribuciones del educando para su sociedad.

Tomando en cuenta las características del teatro y sus actividades dramáticas se pueden explorar y destacar los valores humanos universales en contra posición de los antivalores tales como violencia, irrespeto, menosprecio a la vida, desobediencia, egoísmo en fin carencia de valores y la consecuencia que conlleva la falta de estos. El estudiante como espectador y actor puede decodificar mensajes a través de dramatizaciones y juego de roles y ponerlo en práctica en su vida rutinaria y auto motivarse para hacer uso de los valores tales como el amor, la solidaridad, la verdad, la rectitud, la paz y no violencia. El teatro, y sus actividades aplicadas en forma didáctica en el aula, y fuera de ella, puede influir en el estudiante y modificar sus ideas, emociones y pensamientos, y esto fortalecería la práctica de valores.

El teatro y su práctica en la escuela fortalecen los lazos de amistad y el trabajo en equipo, fomenta la creatividad, la espontaneidad, la imaginación y fortalece la autoestima. Favorece el desarrollo del lenguaje, estimula el ejercicio de hablar en público y estructura mejor el pensamiento para poder expresar ideas, emociones y sentimientos; fomenta el ejercicio de la lectura y la adquisición de hábitos para interactuar dentro de una cultura de valores.(Suárez, 2013). 
Segundo Dario Servan Picon

Gonzáles (2019) en su investigación realizada con adolescentes nicaragüenses del nivel secundario demostró que las estrategias didácticas teatrales por su carácter transversal e interdisciplinario se revelan como un instrumento didáctico eficaz para desarrollar aspectos de las competencias básicas y especialmente las de comunicación lingüística; competencia cultural y artística; competencia social y ciudadana; competencia para aprender a aprender; y competencia en autonomía e iniciativa personal. El objetivo general de este estudio es darle un lugar privilegiado al teatro como herramienta pedagógica para el desarrollo de la audición, la expresión oral y corporal así como también el fortalecimiento de los valores en los estudiantes de educación secundaria de Nicaragua. Sin duda como estrategia metodológica el teatro es un gran aporte en educación, tal como refiere Anlas (2018) a través de una metodología basada en el teatro de títeres, cuyas investigaciones mostraron los beneficios del teatro de títeres en la formación de valores en niños de nivel inicial. Concluye así mismo que los títeres orientados a una estrategia en el aula nos permiten enseñar y practicar valores, explicar contenidos, y desarrollar actividades lúdicas. Al igual Velasco (2016) nos dice que con las obras de títeres estás mostrando tu manera de pensar y vivir, transmitiendo valores de vida, que la otra persona, la que está en el público, lo puede recoger o no, pero algo le llegará.

Por su parte Cruz (2014) en su estudio desarrollado con estudiantes con habilidades especiales, en su método refiere que el juego dramático potencia a los estudiantes y los motiva a expresarse de una manera más libre y espontánea facilitándoles la formación de hábitos observando modelos y situaciones de su entorno. La propuesta es que el teatro debe quedar establecido como una herramienta metodológica para estimular y comprender todo tipos de aprendizajes. Finalmente se considera a la dramatización como actividad globalizadora de aprendizaje para la educación en valores y como herramienta unificadora en el tratamiento de necesidades educativas especiales.

Ciriaco (2019) en su investigación propone la inclusión de la expresión dramática en los planes y diseños curriculares en la escuela para promover y reafirmar los valores y actitudes en los estudiantes, trabajar el drama como escenario para disminuir la timidez y promover el respeto y la tolerancia en aras del fortalecimiento de una sana convivencia escolar. Al respecto Rodríguez (2013) afirma que El teatro es un recurso que la comunidad ofrece y, como en la población general, puede ser un instrumento que favorezca la satisfacción de alguna de las necesidades que tiene el indi- 
Segundo Dario Servan Picon

viduo. Por tanto, el teatro es algo más que una expresión artística, es una actividad que implica relacionarse y desarrollar habilidades sociales en personas que presentan sentimientos de soledad y aislamiento social.

En la tesis doctoral "El teatro como recurso educativo para la inclusión" Gil (2016) hace referencia a la importancia de la autoestima como valor para reducir el rechazo entre iguales, el estudio propone el teatro como metodología activa porque permite rescatar al alumno de la inamovilidad de la carpeta y accionar desde la irrealidad para favorecer nuevos vínculos sociales. Los resultados reflejaron que después del entrenamiento en el teatro de la improvisación disminuyeron los rechazos y aumentaron el nivel de autoestima Los vínculos que se originan después del acercamiento físico como propuesta dramática modifican las expectativas y prejuicios establecidos en los estudiantes.

Ayestas \& Previo, (2014) en su investigación de tipo bibliográfica y documental nos acerca al conocimiento y práctica del teatro destacando que el teatro no solo es una medio para generar cambios en quien lo practica sino también en quien observa y participa como espectador, de ahí la doble utilidad del teatro en la formación de los valores, los entrevistados expertos en teatro y actores también reafirman lo que ya es conocido, por ejemplo que el teatro y su impacto en los jóvenes es positivo los motiva a desarrollar valores, morales y éticos que les permite un mejor desenvolvimiento social y los aleja de situaciones al margen de la ley. En una encuesta realizada en este estudio a jóvenes que practican teatro se observa que los valores como el amor y la paz son los más importantes para ellos coincidiendo con los espectadores. Ellos consideran que son la base para desarrollar los demás valores.

Destacando el teatro en el marco responsabilidad social Aparicio (2009) En su proyecto teatral desarrollado con jóvenes en Murcia España buscaba que los participantes sean capaces de construir su conocimiento en valores a través de un aprendizaje significativo, El teatro como opción y espacio para vivenciar concretamente el aprendizaje de valores es lo que le da a su estudio la significatividad y al estudiante le da la oportunidad de aprender de manera concreta experimentando con el teatro. En esta investigación se promueve y se llega a comprender que la acción teatral es una herramienta para transmitir valores y transformar las conciencias. Este proyecto consiguió los siguientes objetivos superar la tolerancia, conseguir el respeto y educar en valores. 
Segundo Dario Servan Picon

Es indudable el alcance positivo de la práctica dramática en el sistema educativo tal como lo demuestra Lorenzo (2018) en su estudio sobre la dramatización y su influencia en las habilidades sociales realizado con alumnos del nivel secundario de la localidad de ate vitarte Lima. Los resultados concluyen que la aplicación del programa de dramatización influye significativamente en las habilidades sociales en los estudiantes y reducen de en gran proporción las conductas agresivas de los estudiantes. Por su parte Espinoza, (2019) pone en evidencia el valor pedagógico del teatro como recurso integral en el proceso de enseñanza aprendizaje en los estudiantes del nivel secundario de Nicaragua, sus conclusiones se fundamentan en la propuesta pedagógica articulando las demás áreas del currículo del nivel escolar con la práctica teatral. Para García (2017) los resultados obtenidos en su estudio reafirman los alcances de la actividad dramática en educación la explicación de su artículo es una parte de un proyecto más amplio donde enfatiza tres aspectos: las relaciones entre educación y drama creativo, las articulaciones entre el teatro y la ciencia y el uso del teatro para la enseñanza de las ciencias.

Arturo y Pretell, (2017) en su investigación correlativa realizada con niños de nivel inicial en Cerro de Pasco encuentran un nivel alto de relación entre el juego dramático y la socialización, los autores concluyen en que la pedagogía teatral es sustancial en el trabajo de las emociones y su reconocimiento así como la dramatización contribuye a superar dificultades relacionadas a la socialización en el aula y fuera de ella. Estableciendo su estudio con niños de la misma edad León y Luis, (2017) concuerdan que la aplicación de la dramatización como técnica influye significativamente en la autoestima. Sustentados en una investigación de tipo experimental y datos estadísticos concluyen que el 95\% de nivel de confianza en la dramatización como técnica en el desarrollo del autoestima.

Los niños y el desarrollo de las competencias comunicativas asociados al juego dramático es el tema de estudio de Rivero ( 2013) la investigación se basa en actividades y experiencias utilizando la palabra y el movimiento, se concluye finalmente que es posible enriquecer el panorama de la expresión dramática en las aulas. Se reconoce además que la expresión dramática es un importante recurso pedagógico, con implicaciones en todas las áreas del currículum. La investigación y la revisión bibliográfica realizada apuntan a las repercusiones de las distintas modalidades dramáticas sobre las dimensiones física, emocional, intelectual o social, puesto que se 
Segundo Dario Servan Picon

relaciona directamente con otros tipos de expresión, favoreciendo una formación plena del educando.

Ayoví y Castillo, (2019) en sus investigaciones con respecto a las estrategias teatrales nos dice que son primordiales en el desarrollo de destrezas, orales y corporales y mejora el rendimiento escolar de los estudiantes, esta investigación propone el diseño de una guía didáctica de talleres de teatro escolar para trabajar competencias comunicativas. Para Rovira, (2019) existe una conexión entre mente, cuerpo y emociones para fortalecer las relaciones interpersonales a nivel comunicativo y solo será posible si aplicamos estrategias lúdicas basadas en un lenguaje teatral. Tapia, (2016) explica y fundamenta que la dramatización no se ha trabajado con objetivos didácticos, comunicativos y expresivos y se ha descalificado como herramienta pedagógica por dos razones: la primera es el hecho de considerar la dramatización como una actividad meramente lúdica y la segunda es la falta de materiales didácticos de dramatización en las aulas, y la falta de conocimiento y dominio del profesorado en el tema. Sala (2020), aborda el tema de la comunicación en el teatro y concluye que el teatro aporta una gran cantidad de ventajas ayudando entre otras a mejorar la comunicación verbal y no verbal incidiendo básicamente en los aspectos del paralenguaje, kinésicos, y la proxémica. Sin embargo, es necesario considerar que, para un trabajo óptimo el teatro debería trabajarse en forma articulada con el área de comunicación y lengua.

\section{CONCLUSIONES.}

El teatro como propuesta metodológica en el campo educativo es una gran fortaleza para desarrollar actitudes, valores y habilidades en los estudiantes. La expresión dramática más que un producto final, estético y de espectáculo es una actividad cuyo valor intrínseco está en el fomento de la sinceridad, la libre expresión y el aprendizaje de hábitos en base a experiencias vivenciales. El teatro en la escuela es un pilar fundamental para la formación de la persona, más allá de cualquier condición, ya sea su edad o procedencia. Practicar e internarse en los conocimientos del teatro es relacionarse con la historia de nuestra vida. Visto desde este ángulo, el arte teatral integra la historia, la literatura, la sociología, la filosofía"

-Es preciso señalar que el teatro está inmerso en los planes de estudio del actual currículo nacional, sin embargo el teatro en las escuelas continua siendo una actividad que se realiza por el esfuerzo de un reducido grupo de educadores y profesionales voluntarios, la práctica teatral no está generalizado en los centros educativos, en 
Segundo Dario Servan Picon

consecuencia las representaciones suelen ser escasas y en ellas participan una minoría de alumnos escogidos, preparados fuera del horario escolar, La finalidad en este caso es prepararse para un programa en particular. A propósito la Competencia relacionada a la creatividad no dice que el estudiante debe usar los diversos lenguajes artísticos el teatro y otras disciplinas para expresar o comunicar mensajes, ideas y sentimientos. Poniendo en práctica sus habilidades imaginativas y reflexivas para generar ideas, planificar, concretar propuestas y evaluarlas de manera continua MINEDU, (2016)

Integrar el teatro y la dramatización en el aula no es realizar prácticas circunstanciales destinadas a preparar un pequeño espectáculo o amenizar las fiestas del centro educativo, ni el resultado de una improvisación constante o falta de planificación por parte del profesor. Por el contrario, se demanda de un compromiso de IE por reconocer el valor educativo del teatro como recurso para la formación integral de los alumnos, así como la implementación del teatro con una metodología a seguir para introducir este recurso educativo en el aula.

\section{REFERENCIA BIBLIOGRAFICA}

Anlas, M. (2018). teatro de títeres como medio de enseñanza de valores de los niños de 5 años de la i.e.i. $n^{\circ} 602$ - manchego muñoz el tambo - huancayo - 2018. 1-7.

Arnheim, R. (1997). Rudolf Arnheim - Arte y percepción visual (1997, Alianza Forma).

Arturo, G., \& Pretell, R. (2017). “ El juego dramático y la socialización de los niños y niñas del Jardín Elvira García y García. http://repositorio.ucv.edu.pe/bitstream/handle/UCV/26821/rosas_mj.pdf?sequence=1\&i sAllowed $=y$

Ayestas, E. G. T., \& Previo. (2014). El Teatro Como Medio de Comunicación Alternativo para la Transmisión de Valores. 1-86.

Ciriaco, R. E. C. C. F. M. D. A. (2019). La Expresión Teatral Una Estrategia Para Fomentar Los Valores Y Fortalecer El Ambiente Escolar. Society.

Cebrián Velasco, B. (2016). El títere y su valor educativo. Análisis de su influencia en Titirimundi, Festival Internacional de Títeres de Segovia. 354. https://uvadoc.uva.es/bitstream/handle/10324/16249/Tesis838160224.pdf? sequence $=1 \&$ isAllowed $=\mathrm{y}$

Corzo, J. R. F. (2004). Los Valores Y Sus Desafios Actuales. 368. http://books.google.com/books?id=GeokCQ5oRXYC\&pgis=1 
Segundo Dario Servan Picon

Cruz Cruz, P. (2014). El juego teatral como herramienta para el tratamiento educativo y psicopedagógico de algunas situaciones y necesidades especiales de la infancia.

Domínguez, C. del C. D. (2020). El potencial del teatro en educación infantil. 139. https://riull.ull.es/xmlui/bitstream/handle/915/19934/El potencial del teatro en Educacion Infantil.pdf?sequence $=1$

Espinoza, E. J. G. (2019). Aportes pedagógicos del teatro en el proceso de enseñanza aprendizaje en los estudiantes de educación secundaria. https://repositorio.unan.edu.ni/11008/1/11192.pdf.pdf

Fernández Rodríguez, M., Guerra Mora, P., \& Begara Iglesias, O. (2013). El teatro como instrumento terapéutico en la rehabilitación psicosocial. Norte de Salud Mental, XI(46), 82-88.

Frías, S. C. (2009). Teatro y valores en la cultura contemporánea. 1-24.

Gandarillas Hervás, L. (2015). Trabajar la diversidad desde el arte.

García García, J., Parada Moreno, N., \& Ossa Montoya, A. (2017). El drama creativo una herramienta para la formación cognitiva, afectiva, social y académica de estudiantes y docentes. Revista Latinoamericana de Ciencias Sociales, Niñez y Juventud, 15(2), 839-859. https://doi.org/10.11600/1692715x.1520430082016

Garzón, N. (2015). Didáctica del teatro escolar. http://190.57.147.202:90/xmlui/bitstream/handle/123456789/256/Didactica-teatroescolar.pdf? sequence $=1$

Gil, M. (2016). El teatro como recurso educativo para la inclusión. Segovia (España), 202. http://uvadoc.uva.es/bitstream/handle/10324/22080/Tesis1175170112.pdf? sequence $=1 \&$ isAllowed $=\mathrm{y}$

laferriere, G. (S/N). La pedagogía teatral, una herramienta para educar. Recuperado de: https://core.ac.uk/download/pdf/39108274.pdf

La Sexta (2019). Alumno agrede a profesorcon una papelera. Recuperado de: https://www.lasexta.com/programas/arusitys/virales/la-brutal-agresion-de-un-alumno-aun-profesor-con-una-papelera_201912115df0befb0cf2c45df3179ebe.html

León, R., \& Luis, C. (2017). La dramatización como técnica para desarrollar la autoestima en los niños y niñas de 5 años de la Institución Educativa $N^{\circ} 185$ Gotitas de Amor de Jesús" zona H - Huaycán, Ugel 06 - Ate. 128. http://repositorio.une.edu.pe/bitstream/handle/UNE/1255/Tesis Ruth y Ceccilia dic 2017 APA 12 febrero final.pdf?sequence=1\&isAllowed=y 
LesLuthier (2020) La gallinita dijo eureka. Recuperado de:

https:/www.youtube.com/watch?v=ptorPqV7D5s

MINEDU. (2010). OTP Arte.

Ministerio de Educación del Perú. (2016). Programa Curricular de Educación Básica. In Programa Curricular de Educación Secundaria (p. 259). http://repositorio.minedu.gob.pe/handle/123456789/4550

Motos, T. (2009). El teatro en la educación secundaria: fundamentos y retos. Creatividad y Sociedad, Diciembre de 2009 N¹4, 1-35. https: file://C:/Users/raque/Downloads/Teatr-Secundaria_Fundamentos_Retos.pdf

Motos, T. (2014). Psicopedagogía de la Dramatización. 1996, 36.

Motos Teruel, T. (2008). Habilidades de dramatización y evaluación de la creatividad dramática. Expresión y Comunicación Corporal, Para La Educación, Recreación y Calidad de Vida, 2006, 111-126. http://iacat.com/Revista/recrearte/recrearte07/Seccion3/3. CD. Habilidades dramaticas y ev .pdf

Organización de las Naciones Unidas para la Educación, la C. y la C. (2000). Marco de acción para la educación en valores en la pri mera infancia.

Paoli, A. (2010). Arte y educación en valores. Revista Argumentos, 23(62), pp. $13-37$

Parra Ortiz, J. (2003). La educación en valores y su práctica en el aula. Tendencias Pedagógicas, 8, 69-88

Pérez Lorenzo, R. (2018). El arte dramático para la mejora de las habilidades sociales en estudiantes de tercero de secundaria UGEL No 06, Ate - Vitarte, Lima, 2017. Universidad César Vallejo.

http://repositorio.ucv.edu.pe/bitstream/handle/UCV/14834/Pérez_LRD.pdf?sequence=1 \&isAllowed $=\mathrm{y}$

Perú 21 (2019). Profesor golpeó a 18 alumnos por no cumplir con la tarea en Arequipa. Recuperado de: https://peru21.pe/peru/profesor-golpeo-18-alumnos-cumplirtarea-478476-noticia/

Rivero, L. (2013). La expresión dramática en Educación infantil : desarrollo de las capacidades comunicativas a través del juego dramático. 1-64. https://reunir.unir.net/bitstream/handle/123456789/1886/2013_07_01_TFM_ESTUDIO _DEL_TRABAJO.pdf? sequence $=1 \&$ isAllowed $=\mathrm{y}$ 
Segundo Dario Servan Picon

Rodríguez Aparicio, G. (2009). La Educación en Valores a través del teatro. Hekademos: Revista Educativa Digital, 2, 85-98.

Rosa Ilda Jarquin Gonzáles, J. O. D. (2019). Enseñanza del Teatro como herramienta que permita el desarrollo de la expresión oral, corporal, auditiva y el fortalecimiento de los valores en los estudiantes de educación secundaria nicaragüense.

Rovira, L. C. (2019). El teatro marca la diferencia, "Quiero vivir en paz." 3(Novembere), 1-24. https://doi.org/10.1101/843326

Sala, C. R. (2020). El teatro como recurso para desarrollar la comunicación verbal y no verbal en Educación Primaria. Bachelor's Thesis. https://reunir.unir.net/bitstream/handle/123456789/9912/Ros Sala\%2C Cristina.pdf? sequence $=1 \&$ isAllowed $=\mathrm{y}$

Sandra Ayoví Castillo, F., \& Maritza, C. C. N. (2019). El teatro escolar para el desarrollo de la expresión oral y corporal. Diseño de una guía de teatro. 4(1), 75-84. https://doi.org/.1037//0033-2909.I26.1.78

Suárez, M. (2013). El teatro como alternativa para fomentar los valores en preescolares de segundo grado.

Tapia, I. (2016). Dramatización como recurso educativo. Universidad Pública de Navarra, 119. http://academica-e.unavarra.es/bitstream/handle/2454/21394/TFM16MPES-LCL-TAPIA-109418.pdf?sequence=1

Tierno, B. (2007). Valores humanos. In Books Abroad (Vol. 31, Issue 4). https://doi.org/10.2307/40099679

Touriñan, J. M. (2014). Crisis “de” la educación, crisis “en” la educación y crisis de valores. La educación en crisis. Educación y Crisis Económica Actual, 1-21.

Touriñán López, J. (2018). La relación artes-educación: educamos con las artes y hay educación artística común, específica y especializada. Revista Boletín Redipe, 7(12), 36-92. https://revista.redipe.org/index.php/1/article/view/647 